\title{
Time Series Semi-Markov Decision Process with Variable Costs for Maintenance Planning
}

\author{
R. Dawid, D. McMillan \& M. Revie \\ University of Strathclyde, Glasgow
}

\begin{abstract}
Deciding when and how to maintain offshore wind turbines is becoming even more complex as the size of wind farms increases, while accessibility is challenging compared to onshore wind farms. Planning future maintenance actions requires the wind farm operator to consider factors such as the current condition of the turbine, the cost of a given maintenance action, revenue generated by the asset, weather factors and vessel availability. Rather than making case-by-case decisions for each turbine, the approach described in this paper allows the wind farm operators to automate the process of short-to-medium term maintenance planning through application of a Semi-Markov Decision Process (SMDP). The model proposed here is capable of suggesting the cost-optimal maintenance policy given weather forecast, future vessel costs and availability and the current condition of the turbine. Using the semi-Markov approach, allows the user to implement timevarying failure rate. As the model is capable of utilising time-series data, future weather and vessel constraints can be applied depending on the information available to the user at the time, which will be reflected in the optimal policy suggested by the model. The model proposed here facilitates maintenance decision making in wind farms and will lead to cost reduction through more efficient planning. In addition to that, the model can be used to carry out a cost-benefit analysis of using vessels with different properties.
\end{abstract}

\section{INTRODUCTION}

\subsection{Offshore wind}

Reducing the cost of energy is the key for competitiveness of offshore wind. A recent study has shown that O\&M for a single offshore turbine can cost between $£ 250,000-450,000$ per year (GL Garrad Hassan, 2013), which is a significant proportion of the total cost of the project (often up to a third of total cost of energy (Scheu et al. 2012)). In the recent years, a number of tools have been developed in an attempt to reduce the O\&M cost of offshore wind. Examples of such tools include OMCE developed by Braam et al. (2011), which estimates annual O\&M costs and a tool developed by Dinwoodie et al. (2013), which allows to simulate various operating scenarios, both focused on assessing the benefits of different maintenance solutions.

Furthermore, a comprehensive overview of models applied in wind was provided by Hofmann (2011). The vast majority of offshore wind O\&M models are aimed at the pre-operational stages; for example during the planning stage. In the academic domain, very few models, if any, are made for shortto-medium term maintenance decision support. There is a lack of tools which would help operators decide when and how to maintain offshore wind turbines.

There are a number of challenges in maintenance decision making. Firstly, both the short and longterm weather forecasts need to be considered. Accessibility of offshore wind turbines in the North Sea during winter is very low due to stronger winds and high waves; therefore most of the maintenance needs to be carried during the spring to autumn period. Short-term forecasts are used to determine whether the maintenance actions which were planned for the immediate future can be carried out, or will have to be re-scheduled at a later date.

Secondly, assessing the current state of the component based on condition monitoring data can be difficult. Large volumes of condition monitoring data are analysed by the operators; often certain alarm trigger values are set. Once the value is exceeded, the operators are warned there may be a problem with the turbine. Trend analysis can be used for forecasting (García Márquez et al. 2012). Often the methods used in the industry are crude, while the solutions offered by the academia are overly complicated, discouraging their use in real life (Dujardin et al. 2015).

Thirdly, there are a number of logistical issues to be tackled. Vessels, spares and human resources all 
have to be managed efficiently in order to reduce costs. Savings can be found for example through the opportunistic maintenance approach (Ding \& Tian, 2011). Furthermore, it may be the case that a wind farm operator has a number of vessels at their disposal, each with different properties such as wave height limit, speed or capacity, opening a number of logistical options; selecting the cheapest often won't be trivial.

Fourthly, maintenance planners may have to deal with adverse circumstances such as resource shortages (vessel, spare, human) and unexpected failures. Such events may render previous maintenance schedules useless, meaning that the operators need to be able to adapt quickly, while still choosing the costoptimal actions to take.

Finally, the costs of jack-up vessels can be highly variable, which can also be a factor when planning future maintenance.

In general, maintenance strategies can be timebased or condition-based. Researchers proposing time-based strategies (Andrawus et al. 2009), (Kahrobaee \& Asgarpoor, 2013) and (Karyotakis \& Bucknall, 2010) analyse past failure data to devise optimal maintenance or inspection intervals given costs. However, such strategies lack the flexibility to deal with the majority of issues described above.

Condition-based strategies, such as the one proposed by (Byon \& Ding, 2010) and (Besnard \& Bertling, 2010) are more advanced as the decisions are made based on the condition monitoring data, meaning that maintenance can be performed at a time such that the total cost is minimized. The latter approach is more effective but more difficult to model.

Koopstra \& Heijkoop (2015) argue that previous work on models lack an approach that would be integrated (including all the stakeholders' requirements that are necessary to assess the effectiveness of the O\&M) and generic (applicable to all wind farms). The condition-based model proposed in this paper attempts to address most of the issues discussed in this section, bridging the gap between the models proposed by the academia and usability on a day-to-day basis during the operation of a wind farm.

The rest of this paper is organized as follows: Section 2 describes the methodology used in the model, Section 3 provides a case study to illustrate the model outputs before conclusions are summarized in Section 4.

\section{METHODOLOGY}

The basis of this model is a non-stationary Markov Decision Process (MDP). The use of Markov models in the wind energy industry has been reviewed in more detail in (Dawid et al. 2015). MPDs are predominantly used to optimize the offshore wind turbine maintenance, however, most papers in this field use fixed maintenance intervals, which is more applicable to preventative maintenance rather than corrective. One limitation of the majority of applications of MDPs is that costs are fixed over time (i.e. stationary). In reality, the cost of maintenance in some fields, particularly offshore wind, can be highly variable over time, which has a significant impact on the choice of optimal action. In nonstationary MDPs, the assumption that all the inputs used are fixed over time is relaxed.

A survey for research articles (which included the title, abstract and author keywords) containing the phrase "non stationary Markov decision process maintenance" on the Web of Knowledge website ${ }^{1}$ returned 6 results, suggesting that this approach has not been widely applied in the field of maintenance optimization. Of the 6 papers, only 4 were used for optimizing the maintenance schedule, they are discussed below.

Jin et al. (2016) applied partially observable MDPs, which can deal well with the incomplete information coming from the condition monitoring equipment. The methodology allows both nonstationary transition rates as well as variable costs. Papakonstantinou \& Shinozuka (2014) also used partially observable MDPs, however their model only allows non-stationary deterioration and not cost.

Nguyen et al. (2014) employed the MDP methodology for maintenance decision making focusing on the effect of technological advancements on the type of decision taken. The model described by Niese \& Singer (2013) somewhat resembles the approach proposed here, however, the authors admit that their decision matrix is only marginally useful to the user, partly due to large size; whereas application of a similar method to the problem described in Section 3 of this paper yielded a decision matrix capable of providing useful information to the user, which is perhaps due to the nature of the problem.

The non-stationary approach is well suited to the offshore wind turbine maintenance problem as it allows considering both age dependent deterioration and variable costs and rewards as shown in the following section.

\subsection{Model formulation}

The subset of possible actions is denoted as A. Number of states of deterioration is denoted by $\mathrm{S}$. The time horizon for the simulation is denoted by $\mathrm{N}$. A final value $F$ is specified at a time $N+1$ : this represents a reward for the system being in a certain state at the end of the simulation; the better the condition of the component at the end, the higher the reward should be (unless the system will be decom-

\footnotetext{
${ }^{1}$ webofknowledge.com
} 
missioned at the end of the simulation, in which case the rewards for each state should be set to 0 ).

The transition matrix $P$, which has a size of SxNxA, comprises of transition rates which can vary over time, making it a Semi-Markov Decision Process (SMDP). The variable failure rates allow the introduction of a bathtub curve, or other failure rate profiles, which is impossible in a standard MDP.

Weather factors are also considered in this model through the use of wind and wave forecasts. Wind prediction is used to estimate the revenue generated $\mathrm{U}$ - by wind turbine on a given day. Significant wave height forecast is used to determine whether the vessel will be able to access the wind turbine on a given day. If an action cannot be carried out due to vessel constraints, the logistical cost of carrying an action at that time - L - is set to a high enough number to eliminate the possibility of including this option in the optimal policy. Different actions may require different types of vessels; the wave height limit for each vessel can be varied in the model to facilitate this. The costs and rewards of all actions over time are defined in a matrix $\mathrm{R}$ which has a size $\mathrm{SxNxA}$. Matrix $\mathrm{R}$ is a summation of all costs, both vessel and repair/replacement cost, as shown in Equation 1.

$R\left(s_{t}\right)=U\left(s_{t}\right)-C\left(a_{t}\right)-L\left(a_{t}\right)$

Costs of actions $\mathrm{C}$ and logistical costs $\mathrm{L}$ are dependent on time and the type of action taken, while rewards $U$ are dependent on the wind forecast (time dependent) and the state of the wind turbine; a failed turbine will produce no revenue. In case of components such as blades, the age may also have a significant effect on how much power is produced; performance of deteriorated blades was shown to be significantly lower compared to brand new ones (Gaudern, 2014) - this can be easily implemented in the model.

\subsection{Model implementation}

One of the procedures for solving MDPs is through value iteration using Bellman equation (Bellman, 1957). After an initial guess of the value is made, the iterations are repeated until convergence, upon which the value of being in a certain state is known. The Bellman's equation used in the model proposed in this paper, is as follows:

$$
V\left(s_{t}\right)=\max _{a_{t} \in A(s, t)} \sum_{s_{t+1}} P_{s_{t} \rightarrow s_{t+1}}^{a_{t}}\left[R_{s_{t} \rightarrow s_{t+1}}^{a_{t}}+d V\left(s_{t+1}\right)\right]
$$

where $\mathrm{V}$ is the value matrix and $\mathrm{d}$ is the discount factor. Here, the iterations are in fact time steps, the simulation starts at $\mathrm{t}=\mathrm{N}$ and is carried out "backwards". The final value of the asset $\mathrm{F}$ becomes the $\mathrm{V}\left(\mathrm{s}_{\mathrm{t}+1}\right)$ in the first iteration, allowing to calculate the values for each state at time $\mathrm{t}=\mathrm{N}-1$. The following iteration is no longer based on $\mathrm{F}$, but on the value calculated in the previous iteration. As it is assumed that the final value of each state specified by the user is exact, the iterative process to find the converged value of a state used in standard MDP solutions is no longer required. In practice, such assumption is not unreasonable, as the final value of an asset at the end of the simulation would be approximately known by the wind farm operator - the value of a turbine being either in an operational or failed state can be quantified through economic analysis. The iterative process ends when $\mathrm{t}=1$. The resulting matrix $\mathrm{V}$ will be of size SxAxN. Optimal actions yielding maximum value are selected based on matrix $\mathrm{V}$. This will result in an optimal policy of a size $\mathrm{SxN}$, which recommends an action for each state, at each time. The outline of the model is shown in Figure 1.

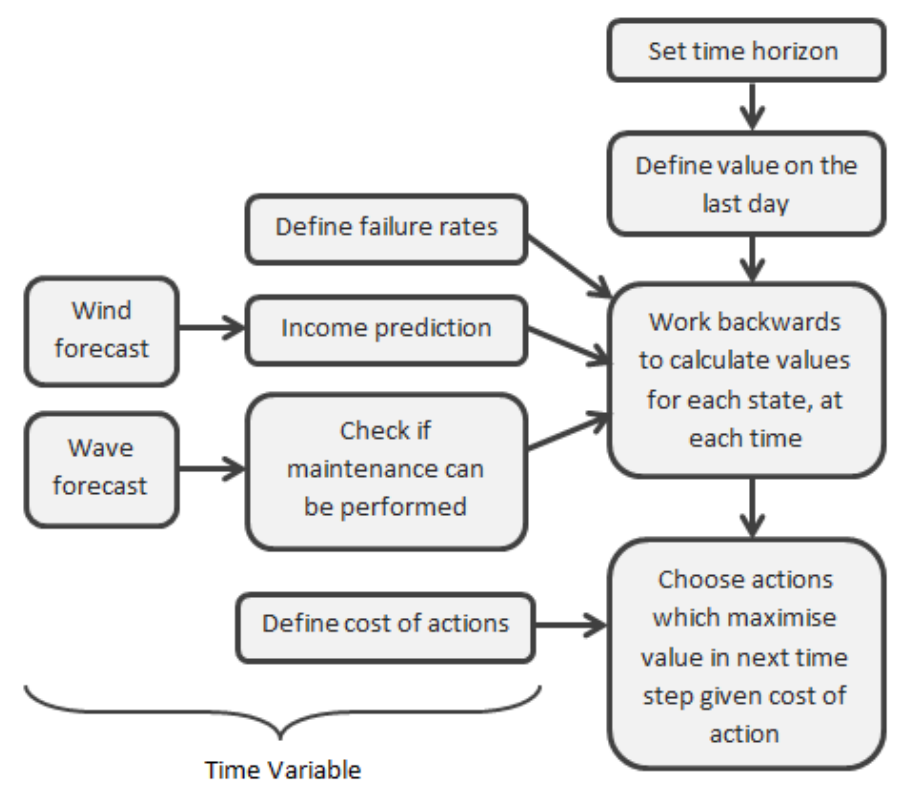

Figure 1. The outline of the model.

\section{CASE STUDY}

\subsection{Model inputs and assumptions}

The methodology described in the previous section was applied to a simple case study based on fictional data. The actions available to the user are to replace, repair and do nothing. Imperfect repairs were used in this case study, meaning that there is a 90\% chance that the component's state will improve by one after repair, $10 \%$ chance it will stay the same. Repair has no effect if the turbine is in a "failed" state. Replacement will always bring the state of the component to brand new condition.

During the "do nothing" action, the system deteriorates according to the defined failure rate, which can, if desired, be time variable. However, for the purposes of this study, it was kept constant, but dependent on the state of the component: $2 \%$ chance of deterioration (per day) from "brand new" to "good", $5 \%$ per day from "good" to "bad", and $15 \%$ chance 
of deteriorating from "bad" to "failed". The "accelerating" failure rate reflects the fact that often by the time a problem is detected through condition monitoring data analysis the time to failure is relatively short.

The costs of repairing and replacing the component were defined, as shown in Table 1.

Table 1. Cost of actions given state (spares/materials).

\begin{tabular}{lcll}
\hline Action cost $\left(£^{`} 000\right)$ & Do nothing & Repair & Replace \\
\hline Brand new & 0 & 5 & 30 \\
Good & 0 & 5 & 30 \\
Bad & 0 & 5 & 30 \\
Failed & 0 & 30 & 30 \\
\hline
\end{tabular}

In addition to the cost of repair itself, the cost of vessel hire can also be significant, particularly when considering the jack-up vessels. In this case study, it is assumed that repairs will be carried out by a Crew Transfer Vessel (CTV), while replacements will require a jack-up vessel. Wind farm operators usually do not own jack-up vessels - they have to be hired from an external company. Often the jack up vessels have to be booked well in advance; short term hires can be very expensive, which was reflected in the inputs to this model, as shown in Table 2.

Table 2. Cost of vessel hire over time.

\begin{tabular}{lcc}
\hline Logistics cost (£`000) & CTV/Repair & Jack-up/Replace \\
\hline Days $1-2$ & 10 & 350 \\
Days 3-9 & 10 & 150 \\
Days 10-12 & 10 & 100 \\
Days 13-15 & 10 & 80 \\
\hline
\end{tabular}

It was assumed that the significant wave height limit below which the vessel can operate is $1.5 \mathrm{~m}$ for CTVs and $2 \mathrm{~m}$ for jack-up vessels. If the either of the vessels limits is exceeded, the cost of action is set to $£ 1,000,000$, effectively eliminating the possibility of it being selected in the optimal policy.

For simplicity, it is assumed that the wind speed is linearly correlated to wave height, with maximum generated revenue (equal to $£ 10,000 /$ day) at wave height equal to 2 meters. The amount generated at other wave heights can be calculated using Equation 3:

$$
U(s, t)=\frac{h * R_{2 m}}{2}
$$

where $R_{2 m}$ is the revenue generated by a turbine when the wave height is 2 meters, $h$ is the wave height. It was assumed that if a repair is carried out, the turbine will only be able to generate $85 \%$ of the revenue it would have generated on that day, if it had not been shut down for repairs. If the state of the wind turbine was "failed", or if the turbine was undergoing a replacement, the revenue generated that day would be $£ 0$. It was assumed that the wind turbine will produce the same amount of revenue, whether the state of the component is "brand new", "good" or "bad".

Time units were set to be days. Discount factor was defined as 0.999 (per unit time, i.e. day). The final rewards at the end of the simulation were set to $£ 150,000, £ 140,000, £ 50,000$ and $£ 0$ for states "brand new", "good", "bad" and "failed" respectively.

A summary of wave height forecast and vessel availability is shown in Figure 2. It allows the operator to see at a glance, on what days the repairs and replacements will not be possible due to wave height constraints.

\begin{tabular}{|l|c|c|c|c|c|c|c|c|c|c|c|c|c|c|c|}
\cline { 2 - 14 } \multicolumn{1}{c|}{} & \multicolumn{11}{c|}{ Time (days) } \\
\hline & $\mathbf{1}$ & $\mathbf{2}$ & $\mathbf{3}$ & $\mathbf{4}$ & $\mathbf{5}$ & $\mathbf{6}$ & $\mathbf{7}$ & $\mathbf{8}$ & $\mathbf{9}$ & $\mathbf{1 0}$ & $\mathbf{1 1}$ & $\mathbf{1 2}$ & $\mathbf{1 3}$ & $\mathbf{1 4}$ & $\mathbf{1 5}$ \\
\hline Wave (m) & 1.1 & 1.2 & 1.3 & 1.4 & 1.5 & 1.6 & $\mathbf{1 . 8}$ & 1.9 & $\mathbf{2}$ & $\mathbf{2}$ & $\mathbf{1 . 7}$ & $\mathbf{1 . 4}$ & $\mathbf{1 . 3}$ & $\mathbf{1 . 2}$ & $\mathbf{1 . 1}$ \\
\hline CTV & $\mathrm{Y}$ & $\mathrm{Y}$ & $\mathrm{Y}$ & $\mathrm{Y}$ & $\mathbf{N}$ & $\mathbf{N}$ & $\mathbf{N}$ & $\mathbf{N}$ & $\mathbf{N}$ & $\mathbf{N}$ & $\mathbf{N}$ & $\mathrm{Y}$ & $\mathrm{Y}$ & $\mathrm{Y}$ & $\mathrm{Y}$ \\
\hline Jack-up & $\mathrm{Y}$ & $\mathrm{Y}$ & $\mathrm{Y}$ & $\mathrm{Y}$ & $\mathrm{Y}$ & $\mathrm{Y}$ & $\mathrm{Y}$ & $\mathrm{Y}$ & $\mathbf{N}$ & $\mathbf{N}$ & $\mathrm{Y}$ & $\mathrm{Y}$ & $\mathrm{Y}$ & $\mathrm{Y}$ & $\mathrm{Y}$ \\
\hline
\end{tabular}

Figure 2. Wave height forecast and predicted vessel availability.

\subsection{Results}

The main output of the model is a set of optimal actions for each time step, for each state of the system, as shown in Figure 3.

\begin{tabular}{|l|c|c|c|c|c|c|c|c|c|c|c|c|c|c|c|}
\cline { 2 - 15 } \multicolumn{1}{c|}{} & \multicolumn{10}{c|}{} & \multicolumn{11}{c|}{} & $\mathbf{1}$ & $\mathbf{2}$ & $\mathbf{3}$ & $\mathbf{4}$ & $\mathbf{5}$ & $\mathbf{6}$ & $\mathbf{7}$ & $\mathbf{8}$ & $\mathbf{9}$ & $\mathbf{1 0}$ & $\mathbf{1 1}$ & $\mathbf{1 2}$ & $\mathbf{1 3}$ & $\mathbf{1 4}$ & $\mathbf{1 5}$ \\
\hline Brand new & 1 & 1 & 1 & 1 & 1 & 1 & 1 & 1 & 1 & 1 & 1 & 1 & 1 & 1 & 1 \\
\hline Good & 2 & 2 & 2 & 2 & 1 & 1 & 1 & 1 & 1 & 1 & 1 & 1 & 1 & 1 & 1 \\
\hline Bad & 2 & 2 & 2 & 2 & 1 & 1 & 1 & 1 & 1 & 1 & 1 & 2 & 2 & 2 & 2 \\
\hline Failed & 1 & 1 & 3 & 3 & 1 & 1 & 1 & 1 & 1 & 1 & 1 & 1 & 3 & 3 & 3 \\
\hline
\end{tabular}

Figure 3. Optimal actions for each state at each time. Action 1 is "do nothing", 2 is "repair", 3 is "replace".

From the optimal policy shown in Figure 3, it can be seen that whenever the system is in the state "brand new" nothing should be done and whenever the system is in a "bad" state, a repair should be carried out (weather permitting).

Optimal actions for states good and failed are more interesting; they are highly dependent on the time due to the variable rewards and costs.

Looking at the second row of Figure 3, it can be seen that the optimal action when in "good" state is to repair on all days before the wave height makes it impossible to use a CTV. At a first glance, it may seem that the most logical policy would be to carry out a repair just before the sea becomes rough (i.e. day 4). However, the optimal policy is right in recommending to repair on days 1,2 and 3 as well, due to the fact that in the model definition, it was specified that the wind speed and therefore the potential revenue generated by the wind turbine is directly proportional to wave height (indicated in Fig- 
ure 2). Repairing in day 1 then makes sense, as the lost revenue due to carrying out a repair would be minimized.

Effectively, the optimal policy's recommendation is to attempt to get the system in the "brand new" state before wave height reaches $1.5 \mathrm{~m}$, as once it does, the cheap option of repair will not be available to the operator for the next week. Once the repair action becomes possible again on day 12, it is no longer essential to be in the "brand new" state, so repairs are only carried out when the system is in a "bad" state, in order to avoid failure, which is expensive.

Looking at the fourth row of Figure 3, the optimal action in a failed state in days 1-2 is to do nothing, which is due to the very high cost of jack-up vessel hire. On day 3, when the cost lowers significantly, it is optimal to replace, however, only until day 4. From then on, it makes more sense to wait to replace until day 13 as this is when the logistical cost is at its lowest. This could be explained by the fact that the difference in logistical cost on days 5 and 13 is more than the revenue the turbine would have generated during the time between days 5-12 (although this is not strictly true, as there is a possibility, albeit small, that despite being repaired on day 5, the turbine would fail again before day 12, which is also considered in this methodology through the use of Bellman equation).

\subsection{Potential model uses}

This model was designed with practical application in mind. It takes seconds to run, is defined by a handful of equations, making it easy for the practitioners to use and understand. There is no constraint on what component the model could be applied to; in fact it would be possible to use the condition monitoring data to classify the condition of the whole turbine, eliminating the need for multiple models.

The model would work well in conjunction with a tool for condition monitoring data analysis, capable of classifying the current condition of the component. One of the possible uses of this methodology could be to forecast future demand for jack-up vessels or CTVs, depending on the predicted future condition of the component, the weather and expected costs of repair. It is possible that further cost savings could be obtained by applying the model to spares management and human resource management.

Suppose the condition monitoring data suggests that a failure, which requires a jack up vessel, will occur in a week's time (day 7 in Figure 3). An instinctive action would be to request a jack-up vessel for day 7 immediately, to avoid further price increase. The resulting cost would be $£ 150,000$ and the income generated by the turbine until the end of the time horizon in this simulation given the expected wind speed would be $£ 63,000$ (days $8-15$, assuming the state remains "brand new"). Instead, if the optimal policy shown in Figure 3 is followed, the cost of jack-up hire will be $£ 80,000$ and the revenue generated $£ 11,500$. Hence the total cost of the first option is $£ 87,000$ compared to $£ 68,500$ for the optimal policy, resulting in a significant cost saving of $£ 18,500$.

As the model's accuracy is highly dependent on the weather forecast, it would be advisable to run it on a daily basis to ensure the most accurate inputs are used. For example, using the policy from Figure 3 , if the condition of the turbine is classified as bad on day 1, the operator knows that if a jack-up vessel will be required in the next 10 days, it would be most cost-effective to hire it for days 3 and 4 .

The case study described in Section 3.1 is a simple one, designed to be easily interpreted. In reality, more states, repair actions and vessel options can easily be considered by the model, allowing it to not only inform the operator when, but also how to maintain the wind turbine.

\section{CONCLUSIONS}

The model presented in this paper has the potential to reduce the cost of offshore wind O\&M by providing decision support to wind farm operators. Resulting policy is optimal as minimizes the total cost, taking into account the vessel constraints, both logistical and repair costs, the lost revenue, current condition of the turbine and forecasted weather conditions.

The novelty of the approach described here is the inclusion of time varying costs and failure rates, which transforms the widely used and relatively simple MDP framework into a useful tool for maintenance decision support.

The model can be used for both short and medium term maintenance planning; it is highly flexible as factors such as time horizon, vessels and their costs and failure rates can be changed easily. As the model is relatively simple and not computationally intensive; it will likely appeal to practitioners.

\section{REFERENCES}

Andrawus, J. a., Watson, J., \& Kishk, M. (2009). Wind Turbine Maintenance Optimisation: principles of quantitative maintenance optimisation. Wind Engineering, 31(0044), 101-110. doi:10.1260/030952407781494467

Bellman, R. (1957). Dynamic Programming. Princeton University Press.

Besnard, F., \& Bertling, L. (2010). An Approach for 
Condition-Based Maintenance Optimization Applied to Wind Turbine Blades. IEEE Transactions on Sustainable Energy, 1(2), 77-83. doi:10.1109/TSTE.2010.2049452

Braam, H., Obdam, T., van de Pieterman, R., \& Rademakers, L. (2011). Properties of the O\&M Cost Estimator (OMCE), (July), 154. Retrieved from http://www.ato.nl/db/WAS4e3a7e8d3ae78/Properties_of _the_O_M_Cost_Estimator_July_2011.pdf

Byon, E., \& Ding, Y. (2010). Season-Dependent ConditionBased Maintenance for a Wind Turbine Using a Partially Observed Markov Decision Process. IEEE Transactions on Power Systems, 25(4), 1823-1834. doi:10.1109/TPWRS.2010.2043269

Dawid, R., Mcmillan, D., \& Revie, M. (2015). Review of Markov Models for Maintenance Optimization in the Context of Offshore Wind, 1-11.

Ding, F., \& Tian, Z. (2011). Opportunistic Maintenance Optimization for Wind Turbine Systems Considering Imperfect Maintenance Actions. International Journal of Reliability, Quality and Safety Engineering, 18(05), 463481. doi:10.1142/S0218539311004196

Dinwoodie, I., McMillan, D., Revie, M., Lazakis, I., \& Dalgic, Y. (2013). Development of a combined operational and strategic decision support model for offshore wind. Energy Procedia, 35, 157-166. doi:10.1016/j.egypro.2013.07.169

Dujardin, Y., Dietterich, T., \& Chad, I. (2015). $\alpha$-min: A Compact Approximate Solver For Finite-Horizon POMDPs, (Ijcai), 2582-2588.

García Márquez, F. P., Tobias, A. M., Pinar Pérez, J. M., \& Papaelias, M. (2012). Condition monitoring of wind turbines: Techniques and methods. Renewable Energy, 46, 169-178. doi:10.1016/j.renene.2012.03.003

Gaudern, N. (2014). A practical study of the aerodynamic impact of wind turbine blade leading edge erosion. Journal of Physics: Conference Series, 524, 012031. doi:10.1088/1742-6596/524/1/012031

GL Garrad Hassan. (2013). A Guide to UK Offshore Wind Operations and Maintenance. Scottish Enterprise and The Crown Estate.

Hofmann, M. (2011). A Review of Decision Support Models for Offshore Wind Farms with an Emphasis on Operation and Maintenance Strategies. Wind Engineering, 35, 1-16. doi:10.1260/0309-524X.35.1.1

Jin, L., Bayarsaikhan, U., \& Suzuki, K. (2016). Optimal control limit policy for age-dependent deteriorating systems under incomplete observations. Proceedings of the Institution of Mechanical Engineers, Part O: Journal of Risk and Reliability, 230(1), 34-43.
doi:10.1177/1748006X15589208

Kahrobaee, S., \& Asgarpoor, S. (2013). A hybrid analyticalsimulation approach for maintenance optimization of deteriorating equipment: Case study of wind turbines. Electric Power Systems Research, 104, 80-86. doi:10.1016/j.epsr.2013.06.012

Karyotakis, A., \& Bucknall, R. (2010). Planned intervention as a maintenance and repair strategy for offshore wind turbines. Proceedings of the Institute of Marine Engineering, Science and Technology Part A: Journal of Marine Engineering and Technology, 27-35.

Koopstra, H., \& Heijkoop, G. (2015). Integrated Decision Support Tool for Planning and Design of Offshore Wind O \& M Strategies. EWEA, 1-5.

Nguyen, T. P. K., Castanier, B., \& Yeung, T. G. (2014). Maintaining a system subject to uncertain technological evolution. Reliability Engineering and System Safety, 128, 56-65. doi:10.1016/j.ress.2014.04.004

Niese, N. D., \& Singer, D. J. (2013). Strategic life cycle decision-making for the management of complex Systems subject to uncertain environmental policy. Ocean Engineering, $\quad 72, \quad 365-374$. doi:10.1016/j.oceaneng.2013.07.020

Papakonstantinou, K. G., \& Shinozuka, M. (2014). Optimum inspection and maintenance policies for corroded structures using partially observable Markov decision Processes and stochastic, physically based models. Probabilistic Engineering Mechanics, 37, 93-108. doi:10.1016/j.probengmech.2014.06.002

Scheu, M., Matha, D., Hofmann, M., \& Muskulus, M. (2012). Maintenance strategies for large offshore wind farms. Energy Procedia, 24(January), 281-288. doi:10.1016/j.egypro.2012.06.110 